\title{
Performance evaluation of MANET routing protocols based on QoS and energy parameters
}

\author{
Salma S. Mohamed, Abdel-Fatah I. Abdel-Fatah, Mohamed A. Mohamed
}

Faculty of Engineering, Mansoura University, Mansoura, Egypt

\begin{tabular}{l} 
Article Info \\
\hline Article history: \\
ReceivedNov 23, 2019 \\
Revised Jan 11, 2020 \\
Accepted Jan 29, 2020 \\
\hline
\end{tabular}

Keywords:

Ad hoc networks

MANET

Network simulation

Performance evaluation

QoS

Routing Protocols

\begin{abstract}
Routing selection and supporting Quality of Service $(\mathrm{QoS})$ are fundamental problems in Mobile Ad Hoc Network (MANET). Many different protocols have been proposed in the literature and some performance simulations are made to address this challenging task. This paper discusses the performance evaluation and comparison of two typical routing protocols; Ad Hoc On-Demand Distance Vector (AODV) and Destination-Sequenced DistanceVector (DSDV) based on measuring the power consumption in network with varing of the QoS parameters. In this paper, we have studied and analyzed the impact of variations in QoS parameter combined with the choice of routing protocol, on network performance. The network performance is measured in terms of average throughput, packet delivery ratio (PDR), average jitter and energy consumption. The simulations are carried out in NS-3. The simulation results show that DSDV and AODV routing protocols are less energy efficient. The main aim of this paper is to highlight the directions for the future design of routing protocol which would be better than the existing ones in terms of energy utilization and delivery ratio.
\end{abstract}

Copyright (C) 2020Institute of Advanced Engineering and Science. All rights reserved.

\section{Corresponding Author:}

Salma Subh Mohammed,

Department of Electrical and Commincation Engineering,

Mansoura University, Egypt.

Email: salma_subh@yahoo.com

\section{INTRODUCTION}

Since 1990, MANET has been a popular research topic and MANETshave also been used in different applications. Supporting mobility of nodes in MANET with hundreds of nodes has been a main challenge in this kind of network, due to limited wireless transmission range, packet losses because of transmission errors, mobility induced route changes, and energy constraints [1].Therefore energy efficiency is important metric for sending the data from source to destination. Routing protocol is used for maximizing the energy efficiency of the network [2, 3]. There are a number of routing protocols for ad-hoc networks [4, 5], they are categorized into Proactive Routing and Reactive routing.Proactive routing protocols or table-driven protocols follow an approach similar to the one used in wired routing protocols. Each node maintains routing table which contains information about the network topology even without requiring it. So, the route in the network is predetermined for example DSDV [6, 7]. Reactive routing or on-demand protocols does not attempt to continuously determine the network connectivity, rout is discovered whenever it is needed. There are various reactive protocols such as AODV $[8,9]$.

The major difference between AODV and DSDV is that DSDV, the source and the intermediate nodes store the neighbor's node information according to each flow for data packet transmission. It generates no more packets for communication. The connection setup delay is lower and it consumes more share of the bandwidth in addition to taking more time to build routes. Intermediate nodes can lead to inconsistent routes. DSDV protocols are not appropriate for large network or highly dynamic as they need to maintainnode entries for each and every node this leads to increased control message overheads which can 
degrade network performance at high loads [10]. Figure 1 and Figure 2 shows the flowchart of AODV and DSDV protocols.

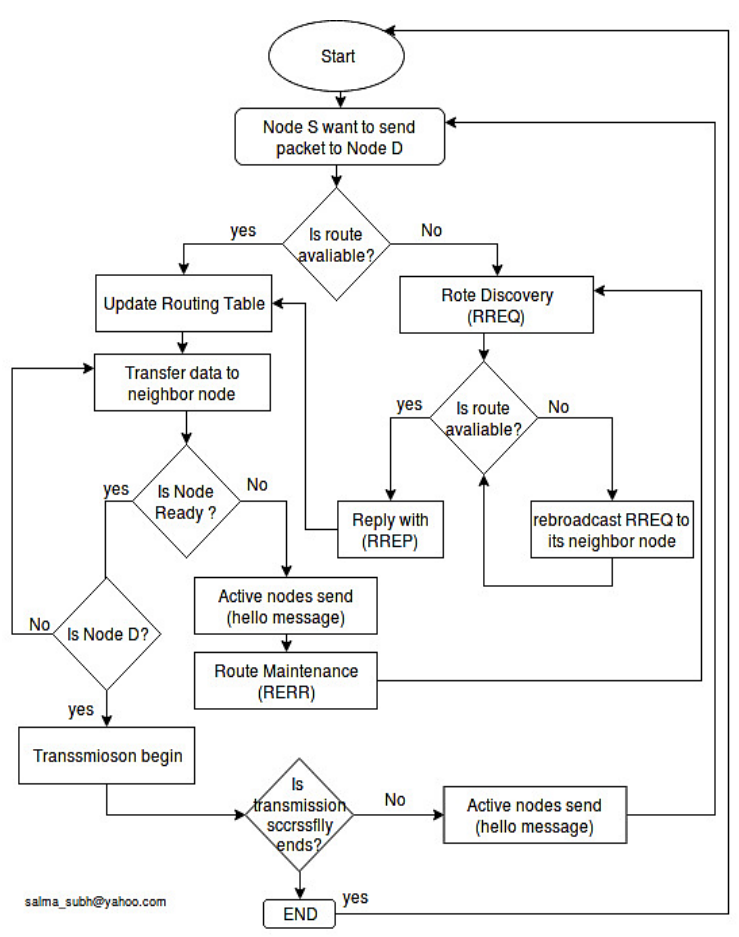

Figure 1. AODV algorithm flow chart

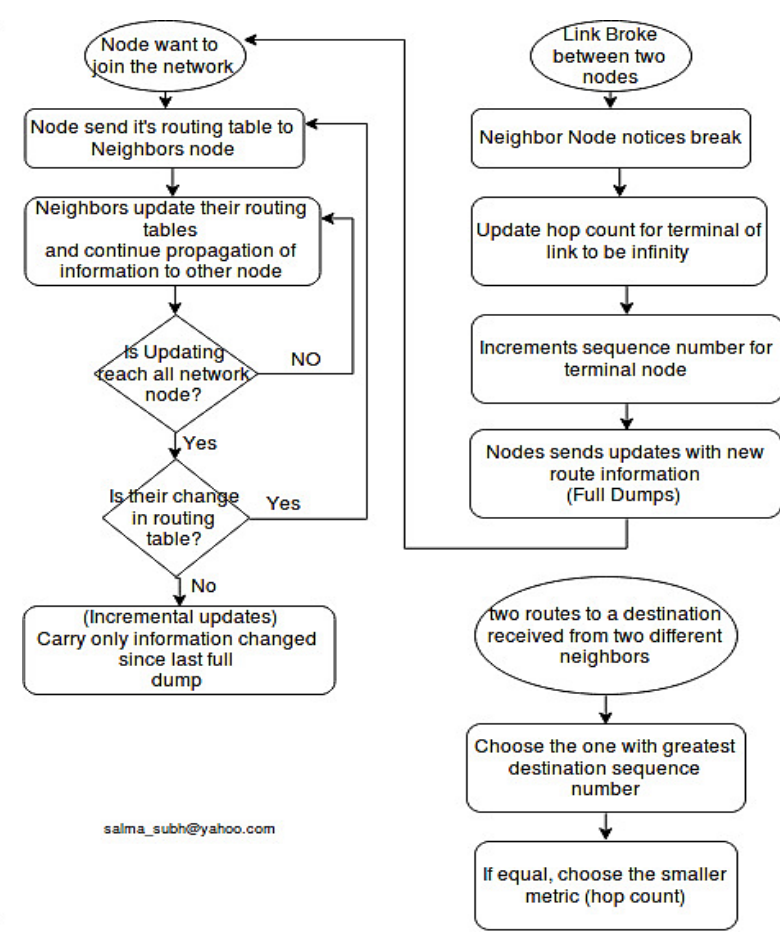

Figure 2. DSDV algorithm flow chart

In recent works, there are a lot of routing protocol have been proposed in MANET which witnessed a huge interest by researchers. The study in [11] has presented a comparison between DSR, AODV and DSDV routing protocols using ns2 simulator to evaluate the performance of these protocols. The results showed that DSDV is the best protocol as compared to AODV and DSR protocols in terms of PDR, packet loss and end to end delay.In [12], the authors have evaluated the performance of DSR, AODV and DSDV routing protocols using NS 2 simulator in terms of the mobility and network load. The simulation results showed that in low mobility and low load scenarios, all three protocols react in a similar way, while with mobility or load increasing DSR outperforms AODV and DSDV routing protocols. This study [13] study impact of mobility patterns on DSR, AODV and DSDV in terms of packet delivery ratio, End to End Delay and average Routing Load. Results shows that DSR is performed the best results in all terms.

In [14], the performance of DSDV, DSR, AODV and OLSR are measured by calculating control overhead, PDR, end-to-end delay and throughput with different number of nodes, different speed (pause time) of nodes and different size of network. The study find that For high mobility condition of nodes DSR gives better packet delivery ratio than other protocols making it suitable for highly mobile random networks. OLSR protocol is the better solution for high mobility condition in large network size with PDR and throughput are the prime criteria. Finally, [15] also compare DSDV, DSR, AODV and OLSR in terms of throughput, packet loss ratio, delays with varying mobility, speed and network load. The result shows DSR should be the first preference in terms of small scale networks with any mobility or speed. AODV or OLSR should be considered when the load of the network is increased. Also many of related works [16-19] do not take into account the energy consumption influence on the protocols, this paper use both QoS and the energy consimuption as the valued metrics in the simulation of MANET routing protocols. We can conclude the aim of our simulation into two main points:

- These studies [20-24] don't take into account the effect on the protocols when the mobile node's pause time is variable or the size of network changing also they donot measure the influence of all these parameter on node energy consumption.

- On other hand, this paper considers the simulation with a dynamic network size and a variable pause time. Hence, performances of the routing protocols investigate under different categories and under various network scenarios. 
The rest of paper is organised as follows. Section 2 presents the research methods of this paper and the simulation environments and the metrics. Section 3 presents the results of simulation. Finally, section 4 presents our conclusions.

\section{RESEARCH METHOD}

This paper emphasizes DSDVand AODV routing protocols, the reasons behind selecting these protocols is that these are the most widely used routing protocols from each of the categories and it was proven that these are the best suited for Ad Hoc Networks. So, a comparison of these two protocols clarifies the general distinction among other protocols of each category. AODV and DSDV are implemented in NS-3 [25]. The main parameters of the simulation are shown in Table 1. The simulation time is 300-seconds (Note that all simulations are started without any established routes in our simulation its take 100 second to create routing table). The random waypoint mobility model was adapted, and the nodes move randomly in the deployment area. After moving to a random target position, there is a pause time before the node starts a new movement.

Table 1. NS-3 parameters setting

\begin{tabular}{cc}
\hline Parameter & Value \\
\hline Network Size & $300 \times 1500 \mathrm{~m}$ \\
Number of Nodes & $50,100,150,200,250$ \\
Number of sources & $20 \%$ of total number of nodes \\
Move Speed & $10,20,30,40,50,60,70,80 \mathrm{~m} / \mathrm{s}$ \\
Pause Time & $0,50,100,300 \mathrm{~s}$ \\
Initial Energy & $100 \mathrm{~J}$ \\
Packet type/Length & CBR (64,128,256,512,1024) bytes \\
\hline
\end{tabular}

The performance of two protocols tested under different scenarios. In one set of scenarios, the value of default parameters is pause time equal to 0 simulation seconds, movement speed is $10 \mathrm{~m} / \mathrm{s}$, the number of nodes is 50 while the number of sources equal to 10 and packet size is $64 \mathrm{k}$ bytes. So, if we examine the impact of movement speed on performances of routing protocols, we vary the maximum movement speed, while the value of other parameters was kept unchanged. Three QoS metrics are used in the simulation, to compare routing protocols performance also; energy consumption and system lifetime are used as comparison parameters. The mathematical equations of these metrics are listed below in the following equations.

$$
\begin{aligned}
& \text { Average Throughput }=\frac{\text { totalnumberof byterecived }}{\text { totaltimeoftransmission }} \\
& \text { End to End Jitter }=\frac{\sum(\text { arrivetime-sendtime })}{\text { totallnumberoftransmitpacket }} \\
& \text { Packet Delivery Ratio }=\frac{\text { totalnumberofrecivedpackets }}{\text { totalnumberoftransmittedpackets }} \\
& \text { Energy Consumption }=N *(\text { initialenergy })-(\text { remainingenergy })
\end{aligned}
$$

System Life Time $=$ It is when $20 \%$ of nodes finish their own batter

\section{RESULTS AND ANALYSIS}

In this section, the comparison has been done for three scenarios the network size (i.e number of node/ sources) to test scalability, mobility pattern (i.e. speed and pause time) and network traffic (i.e. packet size). Energy is a very important factor in routing protocols for MANET because devices may not have the chance to be recharged and so the total energy consumption should be reduced as far as possible, for the two routing algorithms resulting varying one of the four selected parameters i.e. Average Throughputis calculated by (1), End to Enf Jitter is calculated by (2), Packet Delivery Ratio is calculated by (3)Energy Consumptionis calculated by (4) and System Life Time is calculated by (5). 


\subsection{Scenario 1: impact of network size on performance of routing protocols}

In this scenario, the network size varies from 50 to 250 nodes with $20 \%$ of network nodes as a data sources, each source send CBR $64 \mathrm{Kbps}$ packet during the simulation and the results can be concluded as the following:

- A glance on Figure 3 shows that DSDV relatively lower performance than AODV. In detail, when the number of nodes is smaller than 100 nodes, AODV shows the better throughput and energy consumption next DSDV. With the network size bigger than 100 DSDV has the best jitter and PDR next AODV.

- Figure 3.a shows that DSDV has a lower throughput and this is caused by packets that are sent (and lost) before routes have converged initially in the network and due to large amount of routing packet to create the routing table at each node in the network, and along with the increasing number of nodes, AODV also displays the better throughput characteristic.

- Figure 3.b shows that nodes using AODV protocol achieve average jitter higher than DSDV, because AODV is a routing protocol that has no available route when needed it uses the route discovery process. On the other hand, DSDV protocol proactively holds routes to all destinations in its table and this cause high PDR as shown in Figure 3.c, regardless of the density of nodes changes.

- We can observe in Figure 3.d the total energy consumption with varying in network size, the performance of AODV achieve lowest energy consumption during all scenario. Network size greater than 100 nodes consume approximately $85 \%$ of total energy available; on the other hand, with network size close to 250 nodes the node consumes the whole battery at the end of the simulation.

- System lifetime in case of using AODV is approximately $40 \%$ of total operation time of the network, it means that $20 \%$ of nodes finished its battery; otherwise DSDV has long system lifetime since the $20 \%$ lost its power at $56 \%$ of total time. In all scenarios, AODV consumes energy less than DSDV as the results show.

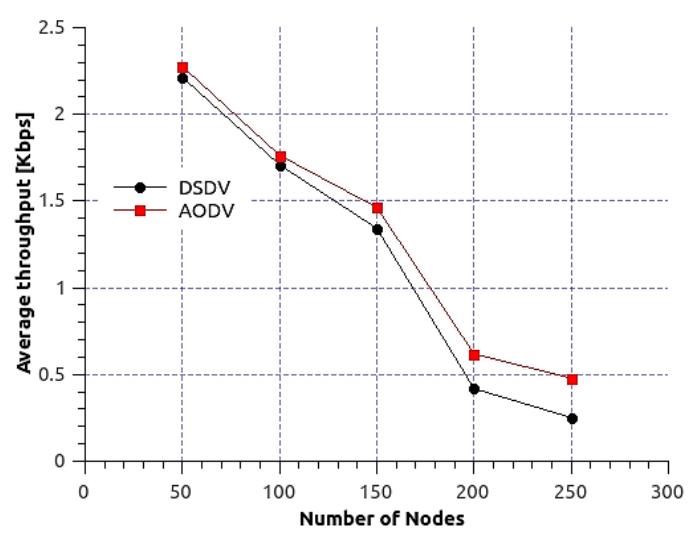

(a)

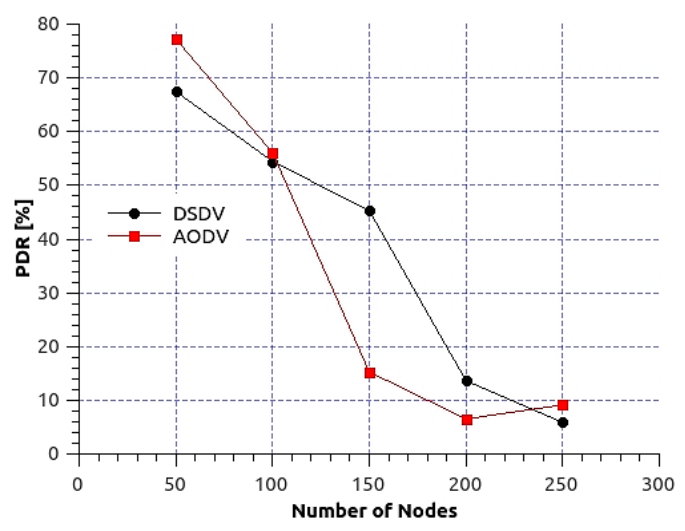

(c)

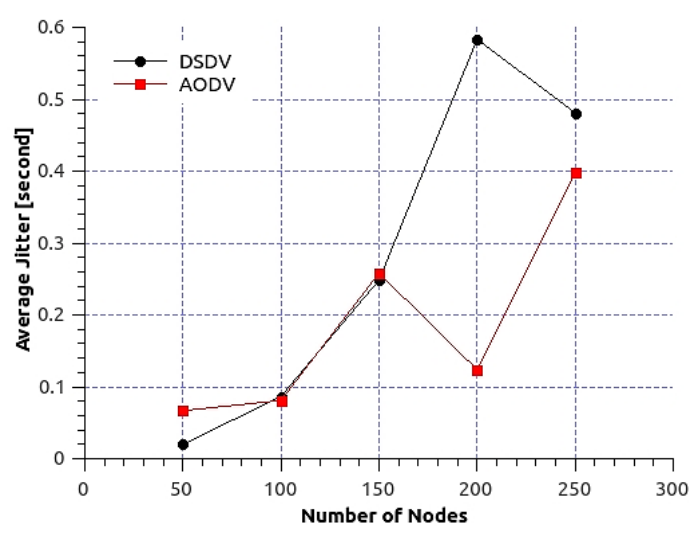

(b)

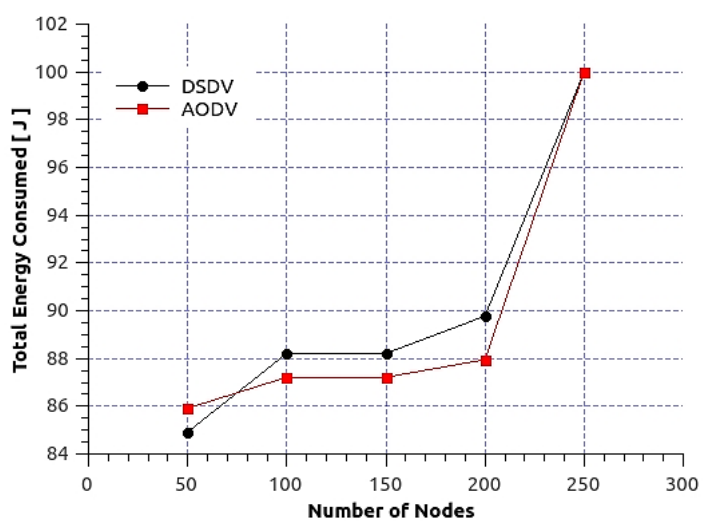

(d)

Figure 3. Network Size impact on performance of routing protocols 


\subsection{Scenario 2: impact of mobility on performance of routing protocols}

Studying the influence of mobility on routing protocol can be achieved through applying two modes of node motion (static and mobile). On simulation, pause time is larger than 200 seconds considering as static mode of node, on other hand, with 0 pause time node move without any pauses with speed equal to $10 \mathrm{~m} / \mathrm{s}$ as shown in Figure 4.a,d also, studying the impact of variation of node speed on routing protocol has been shown in Figure 4.b,c (note during this simulation we sit pause time of node equal to 0 ).

- In Figure 4.a,c, AODV achieves better throughput and PDR at high mobility with varying of node speed up to $80 \mathrm{~m} / \mathrm{s}$ or for small pause time less than 150 seconds. While DSDV has approximately on the contrary at this scenario.

- Since DSDV protocol uses a table-driven approach of maintaining routing information, it is not adaptive to the route changes that occur under high mobility. As a result, DSDV achieves the highest jitter in most of high mobility scenarios as shown in Figure 4.b, because it is slow.

- DSDV consumes less energy than AODV in static mode of mobility as shown in Figure 4.d. in the mobile mode of mobility, due to high changing in topology and increasing in losses. The node in static scenario consumes $80 \%$ of total energy available, while it consumes $100 \%$ of its battery at high speed and small pausing time. It is clear that DSDV more suitable for the static ad-hoc network with a few changes in its topology and its state.

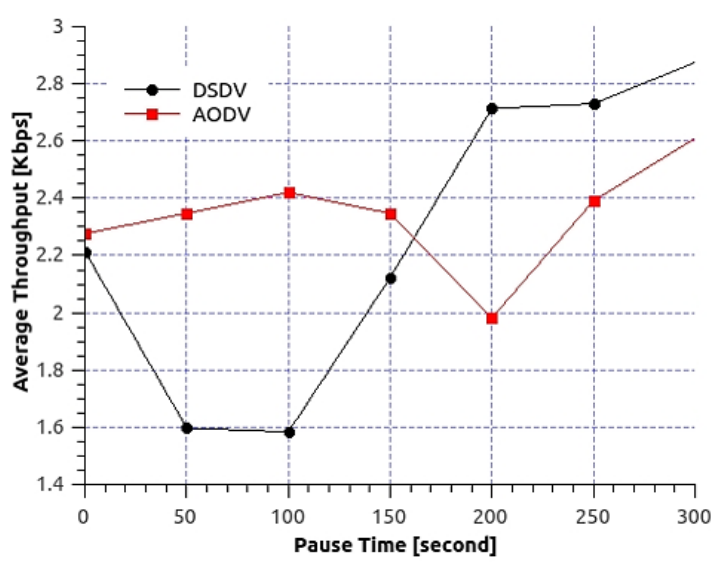

(a)

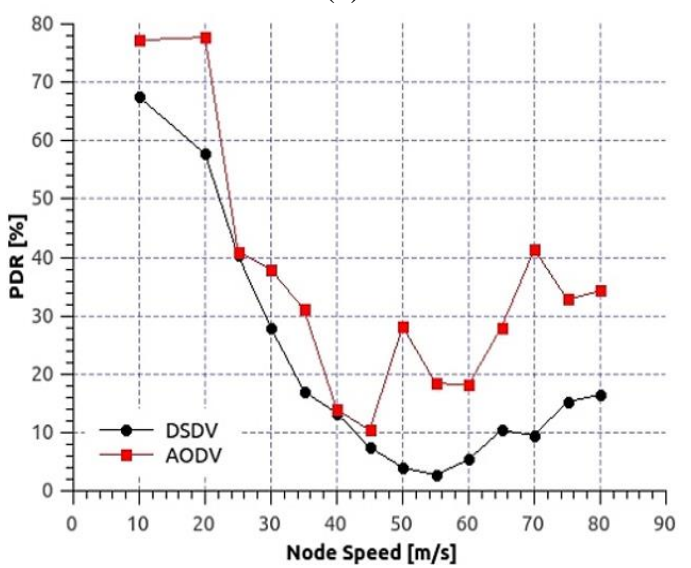

(c)

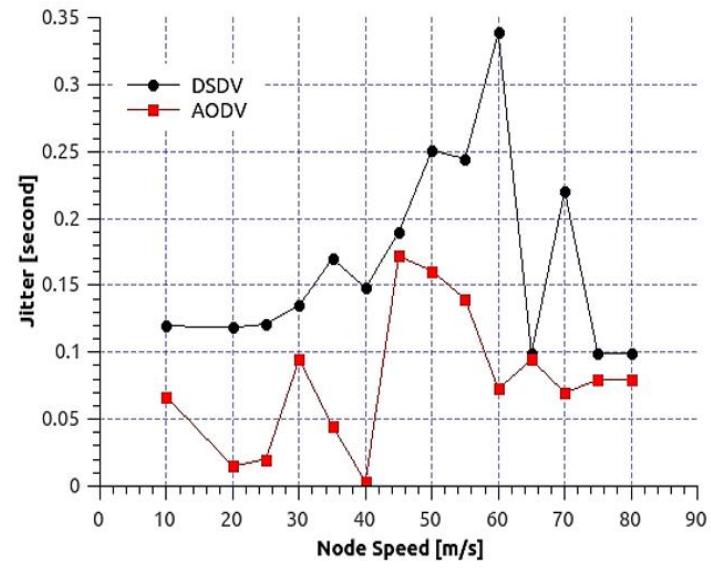

(b)

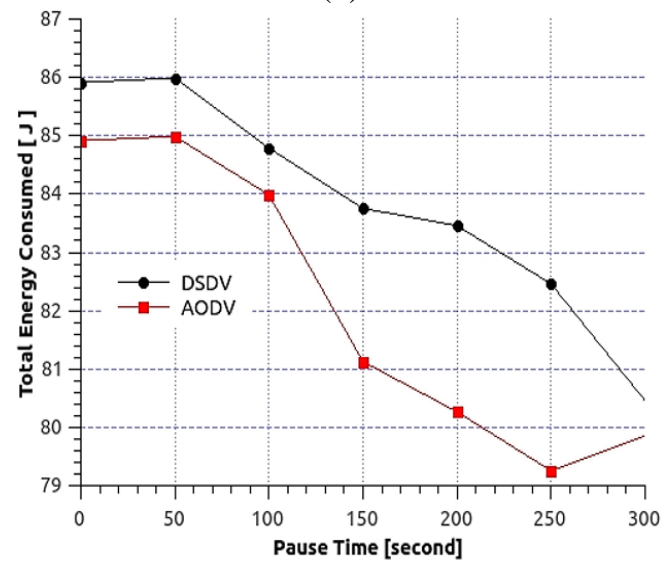

(d)

Figure 4. Mobility impact on performance of routing protocols

\subsection{Scenario 3: impact of packet size on performance of routing protocols}

Traffic effect can be measured by varying the packet size in each scenario, packet size varying from 64 bytes to 1024 bytes CBR data application. The results show in Figure 5 and conclude as follows:

- Generally, throughput decreased for both AODV and DSDV with increased size of packet as shows in Figure 5(a) due to the nature of MANET channel, losses with large packet it's become harder to 
retransmit and lose become painful, but we observe that AODV can deal with losses due to its adaptively for changing and achieve largest PDR than DSDV as shows in Figure 5(c).

- AODV achieves high jitter due to on-demand routing protocol characteristics, as shown in Figure 5(b)

- The consumption in energy at the relatively small size of a packet is approximately constant and the node consumes in average $85 \%$ of its battery as shown in Figure $5(\mathrm{~d})$. The consumption increases with large packet size until reach $90 \%$ of total energy.

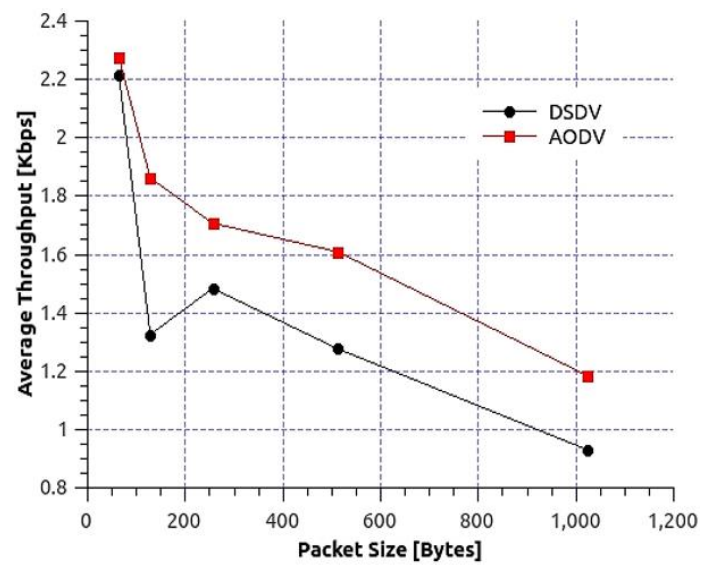

(a)

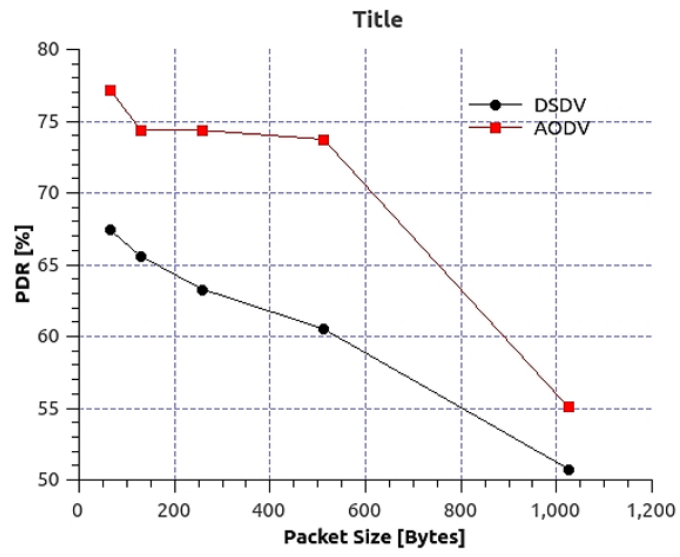

(c)

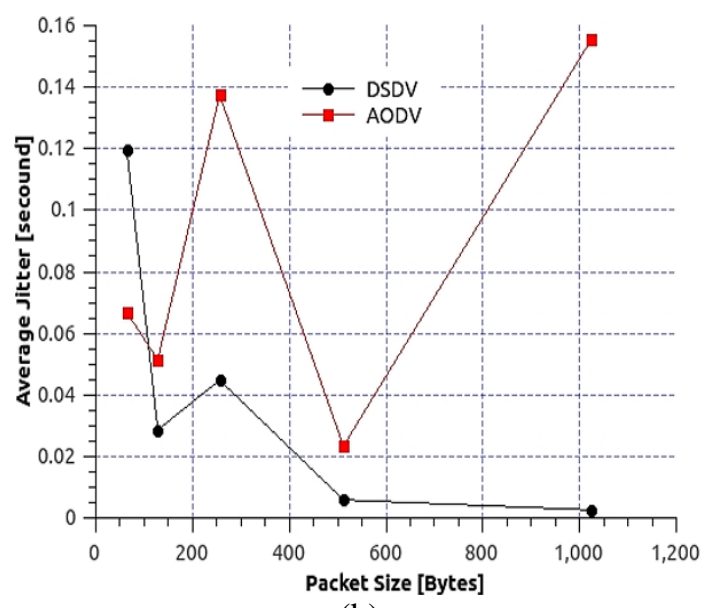

(b)

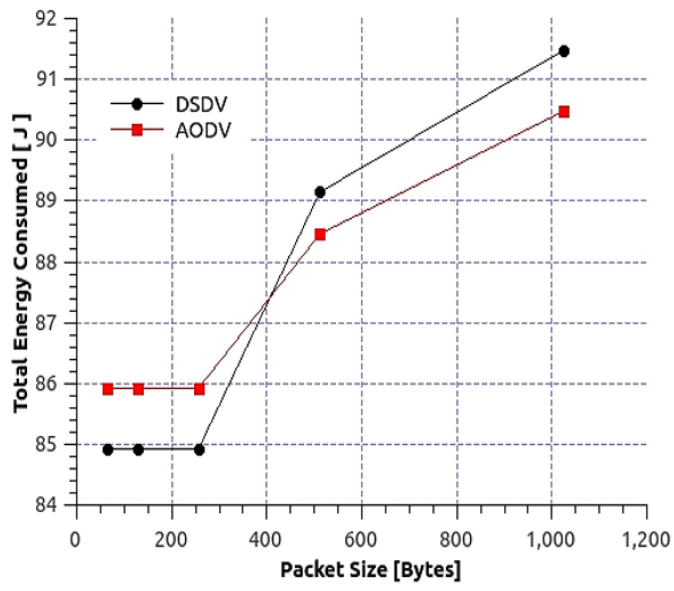

(d)

Figure 5. Mobility impact on performance of routing protocols

The results obtained from the simulations and summarized in Table 2 allow us to conclude to the following. Generally, there is no single protocol performing well under all the performance metrics. As in the simulation results under the different scenarios, the DSDV exhibits attractive performance when the network load and mobility is moderate, while, if heavy traffic and mobility, AODV outperform DSDV and becomes a good candidate to be used.

Table 2. Summary of simulation results

\begin{tabular}{cccccc}
\hline \multicolumn{2}{c}{ Scenarios } & Throughput & Jitter & PDR & Energy consumption \\
\hline \multirow{2}{*}{ Network size } & Small & AODV & AODV & AODV & AODV \\
& large & AODV & AODV & DSDV & DSDV \\
\multirow{4}{*}{ Mobility } & static & DSDV & DSDV & DSDV & AODV \\
& mobile & AODV & AODV & AODV & AODV \\
\multirow{2}{*}{ Packet Size } & Small & AODV & AODV & AODV & AODV \\
& large & AODV & AODV & AODV & DSDV \\
\hline
\end{tabular}




\section{CONCLUSION}

In this paper, two main MANET routing protocols have been evaluated; AODV and DSDV, by varying the selected QoS parameters, i.e. jitter, PDR and average throughput with energy consumption. We generated and simulated three scenarios based on mobility, network size and traffic size. We concluded that the performance of two protocols is more affected while subject to change in mobility pattern as compared to change in the number of nodes. The network load affects directly the performance of protocols and increases the energy consumption on node network.

In the future, there is a need to find an alternative for optimal utilization of power-aware/energyefficient routing in addition to a selection of appropriate energy model. We will use this analysis for enhancing the performance of AODV to decrease energy consumption and increase the lifetime of the network. Also, there is a need to plan to investigate the impact of other applications' traffic (e.g. HTTP, FTP) and transmission protocol (e.g. TCP and UDP) on routing protocols' performance and expand our study towards hybrid routing protocols, considering more metrics and more complex scenarios.

\section{REFERENCES}

[1] C. W. Chen, et al.,"Introduction to the special issue on wireless communication,"IEEE Transactions on Circuits and Systems for Video Technology, vol. 12, no. 6, pp. 357-359, 2002.

[2] D. M. Cordeiro, Carlos, and D. P. Agrawal, "Mobile Ad Hoc Networking,"Center for Distributed and Mobile Computing, ECECS, pp. 1-63, 2002.

[3] I. Chlamtac, M. Conti and J. Liu, "Mobile Ad Hoc Networking: Imperatives and Challenges,"Ad Hoc Networks, vol. 1, no. 1, pp. 13-64, 2003

[4] Alotaibi, Eiman, and B. Mukherjee. "A Survey on Routing Algorithms for Wireless Ad-Hoc and Mesh Networks." Computer Networks, vol. 56, no. 2, pp. 940-965, 2012.

[5] C. S. R. Murthy, B. S. Manoj, "Ad Hoc Wireless NetworksArchitecture and Protocols," Prentice Hall Communications Engineering and Emerging Technologies Series, 2004.

[6] Pathan, Al-Sakib Khan, and C. S. Hong, "Routing in Mobile Ad Hoc Networks," Springer, Guide to Wireless Ad Hoc Networks, pp. 59-96, 2009.

[7] S. Wali, et al.,"A Comprehensive Study on Reactive and Proactive Routing Protocols under Different Performance Metric," Sukkur IBA Journal of Emerging Technologies, vol. 1, no.2, pp 39-51, 2019.

[8] M. K. Marina, and S. R. Das, "Routing in Mobile Ad Hoc Networks", Ad Hoc Networks, pp. 63-90. 2005.

[9] S. Mohseni, et al.,"Comparative Review Study of Reactive and Proactive Routing Protocols in MANETs," In 4th IEEE International Conference on Digital Ecosystems and Technologies, pp. 304-309, 2010.

[10] L. M. Feeney, "A Taxonomy for Routing Protocols in Mobile Ad Hoc Networks", SICS Research Report, 1999.

[11] C.E. Perkins, E.M. Royer, and S.R. Das, "Ad Hoc On Demand Distance Vector (AODV) Routing," IETF Draft, 2000.

[12] F. Mohammed, C. Badr, and E. Abdellah "Comparative Study of Routing Protocols in MANET,"International Conference on Next Generation Networks and Services, pp. 149-153. 2014.

[13] K. Natarajan,and G. Mahadevan. "Mobility based Performance Analysis of MANET Routing Protocols," International Journal of Computer Applications, vol.163, no.10, pp. 37-43, 2017.

[14] B. B. Rao, SK. M. Sharief, and K. GangadharRao. "Impact of Mobility on Routing Protocols in MANET using NS2." Int. Journal of Scientific Research in Computer Science, vol. 3 no.5, pp 120-131, 2018.

[15] S. Baraković, S. Kasapović, and J. Baraković. "Comparison of MANET Routing Protocols in Different Traffic and Mobility Models," Telfor Journal,vol.2, no.1, pp. 8-12, 2010.

[16] N. Sarmah,et al.,"Performance Analysis of MANET Routing Protocols by Varying Mobility, Speed and Network Load," in 9th International Conference IEEESignal Processing and Communication System, pp. 1-6, 2015.

[17] S. Taneja, and A. Kush. "A Survey of Routing Protocols in Mobile Ad-Hoc Networks," International Journal of Innovation, Management and Technology, vol.1 no.3, pp. 279-285, 2010.

[18] R. Bansal, H. Goyal, and P. Singh. "Analytical Study the Performance Evaluation of Mobile Ad Hoc Networks using AODV Protocol,” International Journal of Computer Applications, vol. 14, no.4, pp. 34-37, 2011.

[19] V. K. Quy,et al.,"Survey of Recent Routing Metrics and Protocols for Mobile Ad-Hoc Networks," Journal of Communications, vol. 14, no. 2, pp.110-120, 2019.

[20] F. T. AL-Dhief, et al., "MANET Routing Protocols Evaluation: AODV, DSR and DSDV Perspective," MATEC Web of Conferences EDP Sciences, vol. 150,pp. 1-6, 2018.

[21] S. Mohapatra, and P. Kanungo,"Performance Analysis of AODV, DSR, OLSR and DSDV Routing Protocols using NS2 Simulator,"Procedia Engineering,vol. 30, pp.69-76, 2012.

[22] N. Atri, and R. Goyal,"A Comparative Analysis of MANET Routing Protocols Over TCP," IEEE Control, Instrumentation, Communication and Computational Technologies, International Conference, pp. 712-717, 2015.

[23] A. Sharma, and R. Kumar, "Performance Comparison and Detailed Study of AODV, DSDV, DSR, TORA and OLSR Routing Protocols in Ad Hoc Networks,"Fourth International Conference, Parallel, Distributed and Grid Computing, pp. 732-736, 2016.

[24] M. Rath, B. K. Pattanayak, and B. Pati. "A Contemporary Survey and Analysis of Delay and Power Based Routing Protocols in MANET," ARPN Journal of Engineering and Applied Sciences, vol. 11, no. 1, pp 536-540, 2016.

[25] ns-3, a discrete-event network simulator for internet systems [https://www.nsnam.org/] 


\section{BIOGRAPHIES OF AUTHORS}

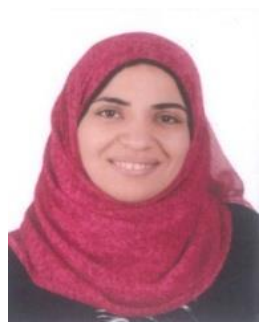

Salma S. Mohamed obtained the B.Sc. degree from the Electronics and Communications Engineering Department in 2011. Hence finished the M.sc. degree in 2015 from Mansoura University and currently preparing for Ph.D. from Mansoura University. She is now an assistant lecture at Mansoura high institute of Engineering and technology.

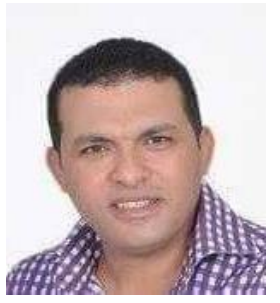

Mohamed A. Mohamed received the Ph.D. degree in Electronics and Communications Engineering from the Faculty of Engineering-Mansoura University-Egypt by 2006. Now he is a professor and dean of Faculty of Engineering, in Mansoura University since 2018. He has 150 publications in various international journals and conferences.

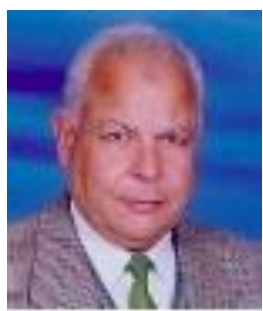

Abdel-Fatah I. Abdel-Fatah was born in Egypt, in, 1941. He received the B.S degree. from Cairo University in 1963 , the M.S. from Assuit University, and the Ph.D degree, from the Technical University of Brno, Czechoslovakia in 1974. All in Electrical Engineering. Currently he is an emeritus professor at the department of electronics and communication engineering Mansoura University, His research interests include; Active circuits, Microelectronics and Nano-electronics. 